\title{
Interocepción y corteza insular: convergencia multimodal y surgimiento de la conciencia corporal
}

Interoception and insular cortex: multimodal convergence and the emergence of body awareness

\author{
Juan Blas Couto ${ }^{1,2,3}$, Lucas Sedeño ${ }^{1}$, Agustín Ibáñez $z^{1,2,3,4^{*}}$ \\ 1 Laboratory of Experimental Psychology and Neuroscience, Institute of Cognitive Neurology, Favaloro University. Buenos Aires, Argentina. \\ 2 Institute of Neuroscience, Favaloro University. Buenos Aires, Argentina. \\ 3 National Scientific and Technical Research Council. Buenos Aires, Argentina. \\ 4 Laboratory of Cognitive and Social Neuroscience, Universidad Diego Portales. Santiago, Chile.
}

\section{Resumen}

La interocepción como censado del estado homeostático y visceral, ha sido recientemente postulada como requisito para la conciencia de estados emocionales corporales (Craig, 2009). Vías parasimpáticas y espinotalámicas que codifican esta información corporal tienen relevo en la corteza insular. Simultáneamente, han sido descriptas otras funciones insulares implicadas en procesos conscientes, como la intencionalidad, la toma de decisiones, la conciencia sensorio-motora, la percepción temporal, reconocer la imagen visual de uno mismo o percibir confiables a otros individuos (Craig, 2009; Ibanez, Gleichgerrcht, \& Manes, 2010). Esta evidencia, sumada a resultados de estudios de lesión y neuroimágenes funcionales, sugiere que la corteza insular anterior (IA) sería la encargada de integrar señales multimodales cognitivas, emocionales y sociales para dirigir las conductas motivacionales que entrañan la supervivencia del individuo. En esta revisión analizamos el cúmulo de evidencia que involucran a la interocepción y al procesamiento insular integrativo en el surgimiento de estados emocionales conscientes, a través de estudios de lesiones y de técnicas de conectividad funcional en resonancia magnética funcional (RMF).

Palabras clave: ínsula, interocepción, conciencia, emociones, cognición social, toma de decisiones

\section{Abstract}

Recent research have suggested that interoception, defined as the sensing of homeostatic and visceral state, is a prerequisite for the emerging of conscious body feelings (Craig, 2009). Parasympathetic and spinothalamic pathways coding such information from the body have their final station in insular cortex neurons. At the same time, several other cognitive functions related to awareness, have been associated with insular activation such as intentionality, decision making, sensory-motor consciousness, time perception, recognition of self's image, or trust on someone else (Craig, 2009; Ibanez et al., 2010). Lesion studies and functional neuroimaging research is in line with this evidence, suggesting that anterior insular cortex would be engaged in integrate multimodal cognitive, emotional and social information in order to modulate motivational behavior leading to the survival of the individual. In this review, we analyze last research works on interoception and integrative insular processing through lesion studies and functional connectivity in functional magnetic resonance imaging (FC-fMRI).

Keywords: insula, interoception, body awareness, empathy, social cognition, decision making

\footnotetext{
* Correspondencia: aibanez@ineco.org.ar. Laboratory of Experimental Psychology and Neuroscience, Institute of Cognitive Neurology (INECO), Pacheco de Melo 1860 (CP 1126), Buenos Aires. Teléfono/Fax: +54 (11) 48120010

Recibido: 14-11-11. Revisión desde: 25-11-11. Aceptado: 28-12-11

DOI: $10.5839 /$ rcnp.2012.0701.06
} 


\section{Introducción}

En los últimos años, sucesivas revisiones subrayan la participación de la corteza insular en múltiples funciones cognitivas (Bechara \& Naqvi, 2004; Craig, 2009; Ibanez et al., 2010) y se ha propuesto que el procesamiento integrativo en la ínsula anterior (IA) podría asociarse a la conciencia subjetiva de las emociones y sentimientos corporales (Craig, 2009). Las evidencias neuroanatómicas de proyecciones de las vías parasimpáticas y termoalgésica espino-tálamo-cortical hacia la corteza insular proponen que estas sensaciones corporales convergen en la ínsula como interocepción. Esta desencadenaría y regularía comportamientos que entrañan un contenido afectivo-emocional esencial para el mantenimiento de la integridad corporal (Craig, 2002). Revisamos aquí evidencia de lesiones y neuroimágenes, proponiendo a la interocepción insular como nexo neural para la coordinación entre el medio interno y el externo, posiblemente implicado en la fisiopatogenia de enfermedades con sintomatología corporal.

\section{Interocepción y la ínsula de Reil}

La interocepción es un sistema neural favorecedor de la homeostasis, que realiza un censado de información visceral (tractos digestivo y genitourinario, aparatos cardiovascular y respiratorio), de receptores vasculares de presión, temperatura y solutos químicos, y nociceptores ubicados en tejidos profundos (músculos y articulaciones) y superficiales (piel) (Craig, 2002).

Una rama parasimpática del sistema nervioso autónomo encargada de mantener un estado restaurativo del organismo y conservador de energía (Porges, 2009) ha sido sugerida como el posible nexo entre los trastornos del ritmo cardíaco y las lesiones insulares, mediante estudios del tono vagal y la variabilidad de la frecuencia cardíaca (HRV, hear rate varability) (Dufey, Hurtado, Fernandez, Manes, \& Ibanez, 2011; Porges, 2009). Por ejemplo, se ha encontrado menor HRV en sujetos post-isquemia insular, indicando regulación parasimpática reducida y aumento en el tono simpático del corazón luego de infartos de la corteza insular derecha. Ello sugiere un desbalance autonómico cortical (Tokgozoglu et al., 1999). Alteraciones electrocardiográficas (Oppenheimer, 2006) y casos de muerte súbita cardíaca han sido relacionados con infartos isquémicos y hemorrágicos de la corteza insular (Abboud, Berroir, Labreuche, Orjuela \& Amarenco, 2006; Cheung \& Hachinski, 2000; Fink et al., 2005).

Por otro lado, la precisión interoceptiva modera significativamente la relación entre cambios de la frecuencia cardíaca y niveles de activación producidos por imágenes emocionales, y esta asociación se incrementa en sujetos con mayor interocepción, como lo demuestra un estudio de análisis del nivel de alerta y de placer reportado ante imágenes emocionales y una tarea interoceptiva de detección de latidos cardíacos (Dunn et al., 2010).

\section{Interocepción y procesamiento del dolor}

El dolor está relacionado con el procesamiento interoceptivo y particularmente la ínsula anterior (IA) parecería jugar un rol de integración supramodal de respuestas autonómicas ante estímulos nociceptivos y de los componentes afectivo- emocionales del dolor (Schnitzler \& Ploner, 2000). Usando resonancia magnética funcional (RMF), Peltz y col. (2011) encontraron que el incremento de actividad simpática asociada a la estimulación dolorosa correlaciona con activaciones en la IA (figura 1.a). Lesiones en la ínsula han sido involucradas con la aparición de síntomas dolorosos, como alodinia e hipoalgesia (Greenspan, Lee \& Lenz, 1999; Moisset \& Bouhassira, 2007), síndrome de dolor central post-isquémico de tipo pseudotalámico y reducción de los efectos y de las reacciones apropiadas ante un estímulo doloroso sin producir ningún cambio de los umbrales de dolor (Schnitzler \& Ploner, 2000). Sin embargo, los pacientes muestran mayor tolerancia y umbrales al dolor producido por frío (vinculados a aspectos afectivosemocionales del dolor), sugiriendo como posible etiopatogenia una falla en la termoregulación interoceptiva. Se sabe que la IA forma parte de la red neural de procesamiento del dolor (Price, 2000), y resultados de conectividad funcional con RMF (Peltz et al., 2011) muestran que en la ínsula posterior-media se producen representaciones interoceptivas de la termoalgesia asociadas al procesamiento térmico discriminativo, mientras que la ínsula anterior participa en la red neural de evaluación de los aspectos afectivo-motivacionales del dolor, apoyando una probable disociación entre ambas dimensiones del dolor (Craig, Chen, Bandy \& Reiman, 2000).

\section{Emociones: el asco y su relación con los sentidos químicos del gusto y el olfato}

Según recientes trabajos, la ínsula procesaría la experiencia y el reconocimiento del asco (Calder, Keane, Manes, Antoun \& Young, 2000; Jabbi, Swart \& Keysers, 2007; Phillips et al., 1997; Sprengelmeyer, Rausch, Eysel \& Przuntek, 1998; Sprengelmeyer, 2007). Sin embargo, reportes de caso único de lesiones insulares muestran resultados divergentes respecto del papel de esta estructura en el procesamiento de señales faciales de asco (Adolphs, Tranel \& Damasio, 2003; Calder et al., 2000; Straube et al., 2010), generando además controversia respecto de la especificidad insular debido a la heterogeneidad de extensión de las lesiones que incluyen zonas córticosubcorticales y sustancia blanca adyacente. Esta limitación orienta futuros trabajos hacia la búsqueda de disociaciones entre déficits asociados a lesiones focales versus daños más extensos, y daños exclusivos versus extrainsulares, apoyados por la localización anatómica de la ínsula y sus múltiples vías de conexión (Augustine, 1996; Mesulam \& Mufson, 1982; Mesulam, Mufson \& Wainer, 1986). Estudios que muestran activación insular conjunta con otras áreas apoyan esta hipótesis (Cauda et al., 2011; Kalani, Kalani, Gwinn, Keogh \& Tse, 2009; Uddin \& Menon, 2009).

El posible rol insular en el procesamiento del asco estaría asociado con el procesamiento sensorial exteroceptivo del gusto y el olfato, evidenciado por experimentos de neuroimágenes que demuestran que la ínsula es sensible a los olores (De Araujo, Rolls, Kringelbach, McGlone \& Phillips, 2003; Frasnelli et al., 2010; Savic \& Gulyas, 2000), y estudios de lesiones insulares reportando alteraciones en la percepción gustativa (Cereda, Ghika, Maeder \& Bogousslavsky, 2002; Pritchard, Macaluso \& Eslinger, 1999; Schafe \& Bernstein, 1998; Small et al., 1999) y en la percepción olfativa (Mak, Simmons, Gitelman \& Small, 2005). La ínsula es considerada el área gustativa primaria (Sewards \& Sewards, 2001; Small et al., 2003) 
y recibe aferencias de la corteza olfativa primaria (Carmichael, Clugnet \& Price, 1994). Esta participación de la IA en circuitos que simultáneamente median la percepción del gusto y la calidad del gusto inducido a través de un olor (Stevenson et al. 2008) sugieren una elaboración gestáltica del sabor en esta corteza (Verhagen, 2007). Wicker y col. (2003) evaluaron la activación insular con RMF al observar expresiones faciales de asco y al sentir olores desagradables, y hallaron activación de IA durante ambas tareas, proponiéndola como sustrato neural común para experimentar asco y reconocerlo en los demás. La integración insular del sabor con el procesamiento emocional específico del asco representaría una ventaja filogenética, debido a que permitiría al individuo inferir que una comida es tóxica y evitar ingerirla al ver en otro una expresión de asco mientras prueba el alimento.

La interocepción insular y la cognición social: empatía, toma de decisiones $y$ adicciones

La empatía por dolor o la habilidad de apreciar las emociones y sentimientos de otra persona en sufrimiento (Decety \& Michalska, 2010) juegan un papel central en el desarrollo moral y de la conducta prosocial (Botvinick et al., 2005; Cheng et al., 2007; Gu \& Han, 2007; Jackson, Meltzoff \& Decety, 2005; Lamm, Nusbaum, Meltzoff \& Decety, 2007; Moriguchi et al., 2007; Saarela et al., 2007; Singer et al., 2004). La empatía por dolor ha sido asociada al procesamiento insular (figura 1.c). Incluso la ínsula pareciera mediar la comprensión del sentido de las acciones (por ejemplo, una persona atacando a otra) a través de sus conexiones prefrontales con áreas temporales y parietales (Amoruso, Couto \& Ibanez, 2011).

Decety y Michalska (2010) examinaron la activación cerebral en sujetos de 7 a 40 años de edad con estímulos disparadores de empatía y demostraron que los más jóvenes califican las situaciones como más dolorosas y poseen mayor activación insular posterior y la red del dolor (figura 2). Esto indicaría mayor nivel de respuestas viscerales ante estímulos dolorosos asociados con un afecto negativo o peligroso, y que a lo largo del desarrollo ontogénico serían reemplazadas por una evaluación más de tipo prefrontal (Swick, Ashley \& Turken, 2008).

El estudio desde la neuroeconomía de las bases neurales de la motivación en la toma de decisiones riesgosas sugiere participación de IA en el procesamiento y aprendizaje de información acerca del riesgo y la incertidumbre (Elliott, Friston \& Dolan, 2000; Grinband, Hirsch \& Ferrera, 2006; Huettel, Stowe, Gordon, Warner \& Platt, 2006; Paulus, Rogalsky, Simmons, Feinstein \& Stein, 2003; Singer, Critchley \& Preuschoff, 2009). Ante respuestas corporales favorecedoras de elecciones adaptativas, la mayor precisión interoceptiva se asocia con mejores decisiones intuitivas (Dunn et al., 2010). Presuchoff y col. (2008) observaron actividad bilateral de la IA durante el cálculo de "predicción del riesgo" y del "error en la predicción", una vez conocido el resultado (figura 1.d). A través de estos dos niveles de representación de la información incierta, la IA puede guiar la toma de decisiones en individuos sensibles al riesgo y modular las tasas de aprendizaje en ambientes inciertos, además de predecir la conducta de riesgo y los errores resultantes (Kuhnen \& Knutson, 2005). Un caso particular de conductas riesgosas son las adictivas; la interocepción insular puede estar relacionada con el procesamiento y aprendizaje de los rituales en el consumo de drogas y su integración en procesos motivacionales explícitos que conducen a la adicción. Se ha demostrado en ratas la interrupción del deseo de consumir después de la desaferentación de la ínsula interoceptiva (Contreras, Ceric \& Torrealba, 2007) y estudios en humanos con lesiones (Naqvi \& Bechara, 2009) y neuroimágenes (Gray \& Critchley, 2007; Naqvi \& Bechara, 2009) confirman estos hallazgos y argumentan acerca del potencial rol de la ínsula en la urgencia de tomar drogas y la interrupción del consumo.
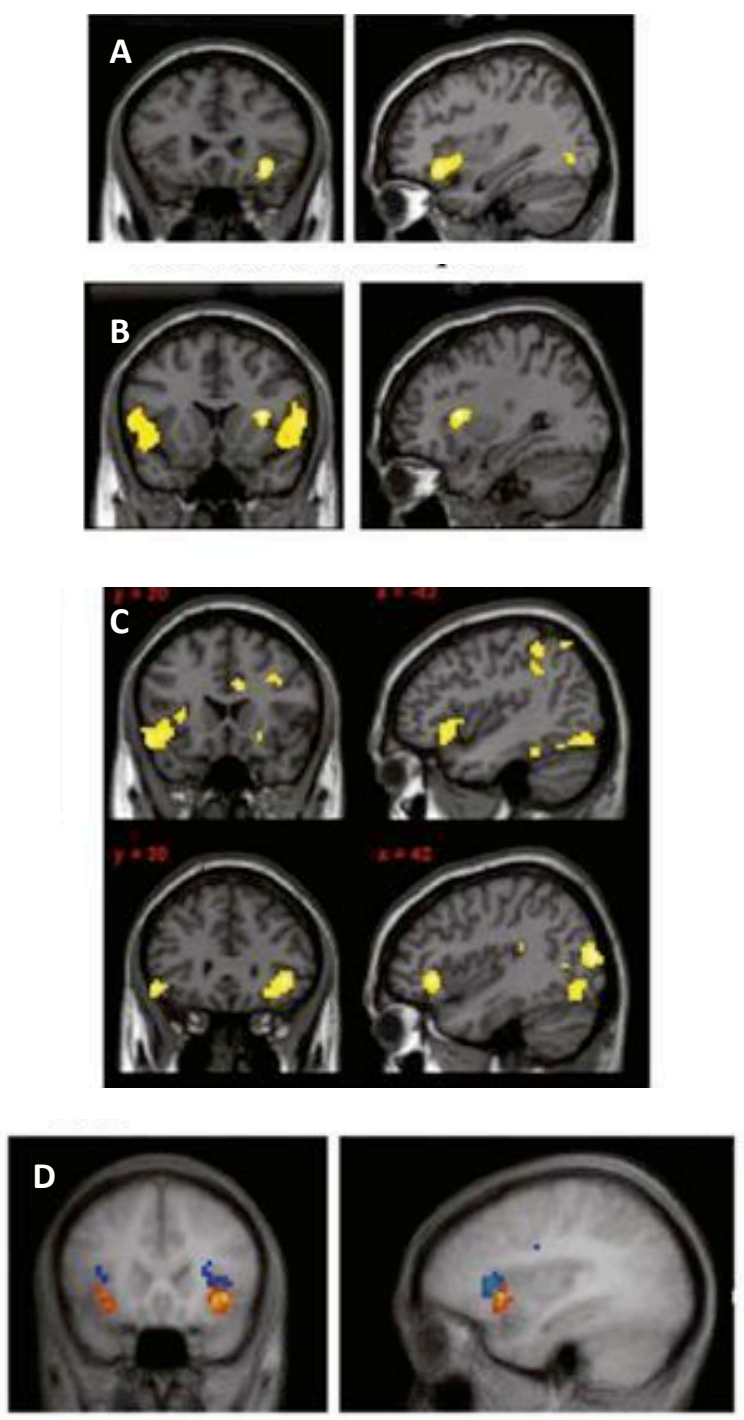

Figura 1. Imágenes de RMF que muestran activación de IA durante activación autonómica, interocepción, empatía y toma de decisiones. Modificado de Singer et al., 2009. A. Activación correlacionada con activación simpática. B. Activación incrementada durante consciencia interoceptiva. C. Aumento de activación al sentir empatía por dolor. D. Predicción de riesgo y predicción del error. 


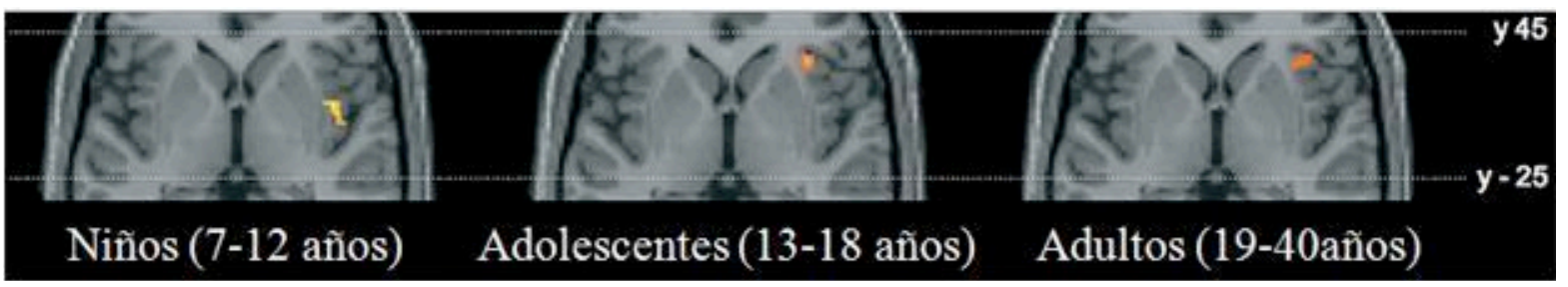

Figura 2. Activación progresiva postero-anterior de la corteza insular ante estímulos de empatía por dolor a lo largo de las edades. Modificado de Decety \& Michalska, 2010.

\section{Discusión}

En suma, la evidencia revisada refleja cómo información visceral y aspectos afectivos del dolor y la temperatura pueden relacionarse con procesos cerebrales de alto orden, tales como el reconocimiento de emociones, la toma de decisiones, la comprensión de acciones o la empatía, al ser procesados en una misma área cortical que es la corteza insular. La IP es la corteza interoceptiva primaria, que recibe aferencias de los núcleos ventromediales (parte posterior, VMpo) del tálamo que codifican información de distintos sistemas de percepción visceral (Craig, 2009; Critchley, 2005). Esta representación de la condición homeostática del cuerpo en la IP ejercería una influencia crucial en el procesamiento cognitivo-afectivo mediante sus conexiones con áreas límbicas y paralímbicas como la amígdala, el hipotálamo y las cortezas cingulada y orbitofrontal (Jackson, Rainville \& Decety, 2006a). De este modo, la interacción entre sensaciones corporales y macroestados cerebrales tendría su sustento en la interocepción, que dirige el comportamiento motivacional (Damasio, 1994; Damasio, 1999) e influencia el procesamiento emocional (Damasio, 1994), la cognición social y la toma de decisiones (Bechara \& Naqvi, 2004; Damasio, 1994).

La relevancia de esta propuesta de asociar la conciencia de estados corporales con procesos cognitivos y conductuales de alto orden es que permite estudiar dentro de un mismo marco situaciones en que uno o ambos se encuentran alterados, como las arritmias cardíacas asociadas a eventos cerebrales, signos de reinervación autonómica postrasplante cardíaco, trastornos de ansiedad con aumento de las señales corporales y trastornos de fisiopatogenia heterogénea como el síndrome del colon irritable y trastornos de la conducta alimenticia. Futuros estudios que aporten mayor evidencia sobre el procesamiento integrativo insular y la relación entre interocepción y distintas variables cognitivas o emocionales serán de gran ayuda en este sentido.

\section{Agradecimientos y conflicto de intereses}

Este trabajo ha sido financiado por becas de CONICET y FINECO.

\section{Referencias}

Abboud, H., Berroir, S., Labreuche, J., Orjuela, K. \& Amarenco, P. (2006). Insular involvement in brain infarction increases risk for cardiac arrhythmia and death. Annals of Neurology, 59, 691-699.

Adolphs, R., Tranel, D. \& Damasio, A. R. (2003). Dissociable neural systems for recognizing emotions. Brain and Cognition, 52, 61-69.

Amoruso, L., Couto, B. \& Ibanez, A. (2011). Beyond Extrastriate Body Area (EBA) and Fusiform Body Area (FBA): Context Integration in the Meaning of Actions. Frontiers in Human Neuroscience, 5, 124.

Augustine, J. R. (1996). Circuitry and functional aspects of the insular lobe in primates including humans. Brain Research Reviews, 22, 229-244.
Bechara, A. \& Naqvi, N. (2004). Listening to your heart: interoceptive awareness as a gateway to feeling. Nature Neuroscience, 7, 102-103.

Botvinick, M., Jha, A. P., Bylsma, L. M., Fabian, S. A., Solomon, P. E. \& Prkachin, K. M. (2005). Viewing facial expressions of pain engages cortical areas involved in the direct experience of pain. Neuroimage, 25, 312-319.

Calder, A. J., Keane, J., Manes, F., Antoun, N. \& Young, A. W. (2000). Impaired recognition and experience of disgust following brain injury. Nat Neurosci, 3, 1077-1078.

Carmichael, S. T., Clugnet, M. C. \& Price, J. L. (1994). Central olfactory connections in the macaque monkey. J Comp Neurol, 346, 403-434.

Cauda, F., D'Agata, F., Sacco, K., Duca, S., Geminiani, G. \& Vercelli, A. (2011). Functional connectivity of the insula in the resting brain. Neuroimage, 55, 823.

Cereda, C., Ghika, J., Maeder, P. \& Bogousslavsky, J. (2002). Strokes restricted to the insular cortex. Neurology, 59, 1950-1955.

Cheng, Y., Lin, C. P., Liu, H. L., Hsu, Y. Y., Lim, K. E., Hung, D. et al. (2007). Expertise modulates the perception of pain in others. Curr Biol, 17, 1708-1713.

Cheung, R. T. \& Hachinski, V. (2000). The insula and cerebrogenic sudden death. Arch Neurol, 57, 1685-1688.

Contreras, M., Ceric, F. \& Torrealba, F. (2007). Inactivation of the interoceptive insula disrupts drug craving and malaise induced by lithium. Science, 318, 655-658.

Craig, A. D. (2002). How do you feel? Interoception: the sense of the physiological condition of the body. Nat Rev Neurosci, 3, 655-666.

Craig, A. D. (2009) How do you feel-now? The anterior insula and human awareness. Nat Rev Neurosci, 10, 59-70.

Craig, A.D., Chen, K., Bandy, D. \& Reiman, E.M. (2000) Thermosensory activation of insular cortex. Nat Neurosci, 3, 184-190.

Damasio, A.R. (1994) Descartes' error and the future of human life. Sci Am, 271, 144.

Damasio, A.R. (1999). How the brain creates the mind. Sci Am, 281, 112-117.

De Araujo, I.E., Rolls, E.T., Kringelbach, M.L., McGlone, F. \& Phillips, N. (2003) Taste-olfactory convergence and the representation of the pleasantness of flavour in the human brain. Eur J Neurosci, 18, 2059-2068.

Decety, J. \& Michalska, K.J. (2010) Neurodevelopmental changes in the circuits underlying empathy and sympathy from childhood to adulthood. Dev Sci, 13, 886-899.

Dufey, M., Hurtado, E., Fernandez, A.M., Manes, F. \& Ibanez, A. (2011) Exploring the relationship between vagal tone and event-related potentials in response to an affective picture task. Soc Neurosci, 6, 48-62.

Dunn, B.D., Galton, H.C., Morgan, R., Evans, D., Oliver, C., Meyer, M. et al. (2010) Listening to your heart. How interoception shapes emotion experience and intuitive decision making. Psychol Sci, 21, 1835-1844.

Elliott, R., Friston, K.J., \& Dolan, R.J. (2000). Dissociable neural responses in human reward systems. J Neurosci, 20, 6159-6165.

Fink, J.N., Selim, M.H., Kumar, S., Voetsch, B., Fong, W.C. \& Caplan, L. R. (2005) Insular cortex infarction in acute middle cerebral artery territory stroke: predictor of stroke severity and vascular lesion. Arch Neurol, 62, 1081-1085.

Frasnelli, J., Lundstrom, J.N., Boyle, J.A., Djordjevic, J., Zatorre, R.J. \& JonesGotman, M. (2010) Neuroanatomical correlates of olfactory performance. Exp Brain Res, 201, 1-11.

Gray, M.A. \& Critchley, H.D. (2007) Interoceptive basis to craving. Neuron, 54, 183186.

Greenspan, J.D., Lee, R.R. \& Lenz, F.A. (1999) Pain sensitivity alterations as a function of lesion location in the parasylvian cortex. Pain, 81, 273-282.

Grinband, J., Hirsch, J. \& Ferrera, V.P. (2006) A neural representation of categorization uncertainty in the human brain. Neuron, 49, 757-763.

Gu, X. \& Han, S. (2007) Attention and reality constraints on the neural processes of empathy for pain. Neuroimage, 36, 256-267.

Huettel, S.A., Stowe, C.J., Gordon, E.M., Warner, B.T. \& Platt, M.L. (2006). Neural signatures of economic preferences for risk and ambiguity. Neuron, 49, 765775.

Ibanez, A., Gleichgerrcht, E. \& Manes, F. (2010) Clinical effects of insular damage in humans. Brain Struct Funct, 214, 397-410. 
Jabbi, M., Swart, M. \& Keysers, C. (2007) Empathy for positive and negative emotions in the gustatory cortex. Neuroimage, 34, 1744-1753.

Jackson, P.L., Meltzoff, A.N. \& Decety, J. (2005) How do we perceive the pain of others? A window into the neural processes involved in empathy. Neuroimage, 24, 771-779.

Kalani, M.Y., Kalani, M.A., Gwinn, R., Keogh, B. \& Tse, V.C. (2009) Embryological development of the human insula and its implications for the spread and resection of insular gliomas. Neurosurg Focus, 27, E2.

Kuhnen, C.M. \& Knutson, B. (2005) The neural basis of financial risk taking. Neuron 47, 763-770.

Lamm, C., Nusbaum, H.C., Meltzoff, A.N. \& Decety, J. (2007) What are you feeling? Using functional magnetic resonance imaging to assess the modulation of sensory and affective responses during empathy for pain. PLoS One, 2, e1292.

Mak, Y.E., Simmons, K.B., Gitelman, D.R. \& Small, D.M. (2005) Taste and olfactory intensity perception changes following left insular stroke. Behav Neurosci, 119, 1693-1700.

Mesulam, M.M. \& Mufson, E.J. (1982). Insula of the old world monkey. I. Architectonics in the insulo-orbito-temporal component of the paralimbic brain. J Comp Neurol, 212, 1-22.

Mesulam, M.M., Mufson, E.J. \& Wainer, B.H. (1986) Three-dimensional representation and cortical projection topography of the nucleus basalis (Ch4) in the macaque: concurrent demonstration of choline acetyltransferase and retrograde transport with a stabilized tetramethylbenzidine method for horseradish peroxidase. Brain Res, 367, 301-308.

Moisset, X. \& Bouhassira, D. (2007) Brain imaging of neuropathic pain. Neuroimage, 37, Suppl 1, S80-S88.

Moriguchi, Y., Decety, J., Ohnishi, T., Maeda, M., Mori, T., Nemoto, K. et al. (2007) Empathy and judging other's pain: an fMRI study of alexithymia. Cereb Cortex, 17, 2223-2234.

Naqvi, N.H. \& Bechara, A. (2009) The hidden island of addiction: the insula. Trends Neurosci, 32, 56-67.

Oppenheimer, S. (2006) Cerebrogenic cardiac arrhythmias: cortical lateralization and clinical significance. Clin Auton Res, 16, 6-11.

Paulus, M.P., Rogalsky, C., Simmons, A., Feinstein, J.S. \& Stein, M.B. (2003) Increased activation in the right insula during risk-taking decision making is related to harm avoidance and neuroticism. Neuroimage, 19, 1439-1448.

Peltz, E., Seifert, F., De Col, R., Dorfler, A., Schwab, S. \& Maihofner, C. (2011) Functional connectivity of the human insular cortex during noxious and innocuous thermal stimulation. Neuroimage, 54, 1324-1335.

Phillips, M.L., Young, A.W., Senior, C., Brammer, M., Andrew, C., Calder, A.J. et al. (1997). A specific neural substrate for perceiving facial expressions of disgust. Nature 389, 495-498.

Porges, S.W. (2009) The polyvagal theory: new insights into adaptive reactions of the autonomic nervous system. Cleve Clin J Med, 76, Suppl 2, S86-S90.

Preuschoff, K., Quartz, S.R. \& Bossaerts, P. (2008) Human insula activation reflects risk prediction errors as well as risk. J Neurosci, 28, 2745-2752.

Price, D.D. (2000) Psychological and neural mechanisms of the affective dimension of pain. Science, $288,1769-1772$

Pritchard, T.C., Macaluso, D.A. \& Eslinger, P.J. (1999) Taste perception in patients with insular cortex lesions. Behav Neurosci, 113, 663-671.

Saarela, M.V., Hlushchuk, Y., Williams, A.C., Schurmann, M., Kalso, E. \& Hari, R. (2007) The compassionate brain: humans detect intensity of pain from another's face. Cereb Cortex, 17, 230-237.

Savic, I. \& Gulyas, B. (2000) PET shows that odors are processed both ipsilaterally and contralaterally to the stimulated nostril. Neuroreport, 11, 2861-2866.

Schafe, G.E. \& Bernstein, I.L. (1998) Forebrain contribution to the induction of a brainstem correlate of conditioned taste aversion. II. Insular (gustatory) cortex. Brain Res, 800, 40-47.

Schnitzler, A. \& Ploner, M. (2000) Neurophysiology and functional neuroanatomy of pain perception. J Clin Neurophysiol, 17, 592-603.

Sewards, T.V. \& Sewards, M.A. (2001) Cortical association areas in the gustatory system. Neurosci Biobehav Rev, 25, 395-407.

Singer, T., Critchley, H.D. \& Preuschoff, K. (2009) A common role of insula in feelings, empathy and uncertainty. Trends Cogn Sci, 13, 334-340.

Singer, T., Seymour, B., O'Doherty, J., Kaube, H., Dolan, R.J. \& Frith, C.D. (2004) Empathy for pain involves the affective but not sensory components of pain. Science, 303, 1157-1162.

Small, D.M., Gregory, M.D., Mak, Y.E., Gitelman, D., Mesulam, M.M. \& Parrish, T. (2003) Dissociation of neural representation of intensity and affective valuation in human gustation. Neuron, 39, 701-711.

Small, D.M., Zald, D.H., Jones-Gotman, M., Zatorre, R.J., Pardo, J.V., Frey, S. et al. (1999) Human cortical gustatory areas: a review of functional neuroimaging data. Neuroreport, 10, 7-14.

Sprengelmeyer, R. (2007) The neurology of disgust. Brain, 130, 1715-1717.

Sprengelmeyer, R., Rausch, M., Eysel, U.T. \& Przuntek, H. (1998) Neural structures associated with recognition of facial expressions of basic emotions. Proc Biol Sci, 265, 1927-1931.
Straube, T., Weisbrod, A., Schmidt, S., Raschdorf, C., Preul, C., Mentzel, H.J. et al. (2010). No impairment of recognition and experience of disgust in a patient with a right-hemispheric lesion of the insula and basal ganglia. Neuropsychologia, 48, 1735-1741.

Swick, D., Ashley, V. \& Turken, A.U. (2008). Left inferior frontal gyrus is critical for response inhibition. BMC Neurosci, 9, 102.

Tokgozoglu, S.L., Batur, M.K., Top Uoglu, M.A., Saribas, O., Kes, S. \& Oto, A. (1999) Effects of stroke localization on cardiac autonomic balance and sudden death. Stroke, 30, 1307-1311.

Uddin, L.Q. \& Menon, V. (2009) The anterior insula in autism: under-connected and under-examined. Neurosci Biobehav Rev, 33, 1198-1203.

Verhagen, J.V. (2007) The neurocognitive bases of human multimodal food perception: consciousness. Brain Res Rev, 53, 271-286.

Wicker, B., Keysers, C., Plailly, J., Royet, J.P., Gallese, V. \& Rizzolatti, G. (2003) Both of us disgusted in My insula: the common neural basis of seeing and feeling disgust. Neuron, 40, 655-664. 